\title{
Topology Smoothing for Segmentation and Surface Reconstruction
}

\author{
Pierre-Louis Bazin and Dzung L. Pham \\ Johns Hopkins University, Baltimore, USA, \\ pbazin1@jhmi.edu, \\ http://medic.rad.jhmi.edu/
}

\begin{abstract}
We propose a new method for removing topological defects in surfaces and volumes segmented from medical images. Unlike current topology correction approaches, we define a smoothing operator that acts solely on the image volume and can be integrated into segmentation procedures. The method is based on an analysis of the scalar field underlying the isosurface of interest, and performs only local changes. No assumptions are required on the structure to segment, or on the desired topology. We show that segmentation algorithms that incorporate toplogical smoothing produce results with fewer topological defects.
\end{abstract}

\section{Introduction}

Topological considerations are a central element in the problem of reconstructing the geometric surface of an anatomic tissue from medical images. Most of these objects have a very simple topology, usually the topology of a sphere. For instance, cortical surfaces despite their intricate geometry, are typically assumed to be topologically spherical. However, surfaces obtained from unconstrained image segmentation are generally not consistent with the anatomical topology: isosurfaces extracted from the segmented volumes often produce large numbers of topological handles due to small errors in the original segmentation.

Mainly for the purpose of cortical reconstruction, recent works have addressed the problem of correcting the defects on the volume or the surface obtained [13 4|12] until spherical topology is reached. These methods act on geometric information alone by placing cuts and filling holes in the surface (or a binary representation of the surface) with little regard to whether this is reflected in the actual data. Alternatively, other strategies start from a topologically correct surface model and then deform it with topologypreserving transformations to perform the segmentation [8,15]. The main drawback of this approach is that finding an initial surface close enough to converge to the desired object becomes a problem with complex geometric shapes.

In this paper, we propose a "topology smoothing" algorithm that locally regularizes surface topology. The technique removes small topological defects while keeping major structures unchanged. No requirements are made on the surface to start from, or on the correct topology to obtain. We also show how the algorithm can be incorporated into a tissue classficiation algorithm to improve the topological consistency of segmentation results. Because it does not impose a hard constraint on the segmentation, it allows deviations from topological regularity where it is deemed appropriate. If a hard topological 
constraint is desired, then the previously described topology correction approaches [13, 4.12] can still be used. Because the topology has already been regularized, fewer cuts and merges will be required.

This paper is organized as follows. We first analyze the link between image segmentation and surface topology, and design a smoothing algorithm from the analysis (Sec,2). That algorithm is then integrated into any tissue classification technique based on fuzzy clustering $(\mathrm{Sec}, 3$. It is validated on cortical segmentation and reconstruction examples (Sec, 3.3).

\section{Smoothing the Topology of Scalar Fields}

\subsection{Duality Between Scalar Fields and Surfaces}

The topology of surfaces is characterized globally by its Euler Number. This measure, however, does not account for the type or extent of local topology changes. Let us consider a surface as the zero level set of a 3D scalar field (for instance, the field of distances to the surface). The surface is in turn recovered from the field as an isosurface (using marching cubes [7,9]). It follows that the surface and the scalar field are two complementary descriptions.

We should expect changes in the surface topology to reflect changes in the field, and vice versa. Indeed, if random noise is added to the scalar field, many small-scale topology changes will occur in the surface. This is the main problem of surface reconstruction from medical images, where some of the noise is propagated into the segmentation, and introduces many topological defects. Smoothing the field smooths the geometry of the associated surface, and should also "smooth" its topology, i.e. remove those topology defects.

\subsection{Critical Points and Topological Variations}

In scalar fields, topology changes occur only at critical points [6 14]. These points are locally the singular points of the implicit isosurfaces, an extension of the non-simple points of binary images [13,5]. They have been classified in [14] as regular, flat, minimal, maximal and saddle points, either local or extended. At any non-regular point, or nonregular region made of extended non-regular points, topology changes for the isosurface can occur when the isovalue used to construct the surface is equal to the value at that point.

If a field has many non-regular points, its topology is likely to be complicated, and to change drastically if the isovalue is modified. It results in a "topological noise", as the topology of the isosurface is unstable for small changes in the isovalue. Following this idea, a field with mostly regular points will be "topologically smooth", i.e. its topology will change only at a small number of isovalues. It is our goal here to produce such smooth topologies: segmented tissues should have a regular topology, wherever the segmentation threshold is. 


\subsection{Classification of Topological Types}

To identify critical points, let us consider a point $X$ with value $f(X)$. We define as positive, negative and equal neighbors the points $Y$ in a neighborhood of $X$ with respectively $f(Y)>f(X), f(Y)<f(X)$ and $f(Y)=f(X)$. With an appropriate neighborhood, the number of positive, negative and equal regions determines the type of the point. We will refer to this type as the topological type of the point 1 . Appropriate neighborhoods here are the smallest possible regions around $X$ with connected neighbors $Y$ [14]. In a discrete field, it is the 6,18 or 26 neighbors of a voxel in a cubic 3D volume, with the connectivity rules usual to volume or surface extraction from digital images [13.5] (however, the connectivity rules don't affect much the smoothing presented here).

Table 1. Topological types and neighbors: $N_{p}, N_{n}, N_{e}$ are the numbers of positive, negative and equal regions in the neighborhood of $X,\langle\cdot\rangle,\langle\cdot\rangle^{+},\langle\cdot\rangle^{-}$are the mean values over the complete neighborhood, the positive regions or the negative regions, respectively.

\begin{tabular}{|c|c|c|c|c|c|}
\hline \multirow{2}{*}{$\begin{array}{c}\text { Topological } \\
\text { type }\end{array}$} & & & & \multirow[b]{2}{*}{ Saddlc } & \\
\hline & Minimum & Maximum & Rcgular & & Flat \\
\hline \multirow[t]{2}{*}{ Conditions } & $\begin{array}{l}N_{p}=1 \\
N_{n}-0\end{array}$ & $\begin{array}{l}N_{p}=0, \\
N_{n}-1\end{array}$ & $\begin{array}{l}N_{p}=1, \\
N_{n}-1\end{array}$ & \begin{tabular}{|c|}
$N_{p}>1$ \\
or $N_{n}>1$
\end{tabular} & $\begin{array}{l}N_{p}=0, \\
N_{n}=0\end{array}$ \\
\hline & \multicolumn{5}{|c|}{ Local: $N_{e}=0$, Extended: $N_{e}>0$} \\
\hline $\begin{array}{c}\text { Topological } \\
\text { neighbor } f^{T}(X)\end{array}$ & $\langle f(Y)\rangle^{\dagger}$ & $\langle f(Y)\rangle$ & $\langle f(Y)\rangle$ & $\begin{array}{l}\langle f(Y)\rangle_{\text {(goin) }} \\
\langle f(Y)\rangle_{\text {(split) }}^{-}\end{array}$ & $f(X)$ \\
\hline
\end{tabular}

Table 1 lists all the possible types, both in 2D and 3D, and the necessary conditions to discriminate between them. This classification is sufficient to detect all topological changes [14]: surfaces appearing and disappearing at maxima and minima, and joining at saddles. The topological type of points will change if and only if its value goes above or below the closest values of its neighbors. Depending on the topological type, we define topological neighbor $f^{T}(X)$ for the value of $X$, as listed in Table 1

For a minimum, a maximum or a saddle point, replacing $f(X)$ with the topological neighbor will force its topological type to change. Saddle points can be affected in two ways: either we lower the value to its negative neighbors value, or raise it to its positive neighbors value. For the underlying surface, it corresponds to "split" or "join" operations. Both choices are valid, so we have to select the most appropriate (usually, the closest value). Flat points are just kept unchanged. Regular points are those we want to reinforce. If a point is regular but close to its above or below neighbors, it will change its topological type with a small perturbation. So to ameliorate the regularity of the point, we place it at a central value.

\footnotetext{
${ }^{1}$ Rigorously, it is the geometric type of the field itself seen as an hyper-surface; we abuse of the topology term to avoid confusing the geometries of the isosurface of interest and of the associated field.
} 


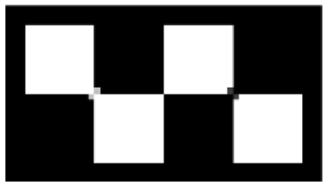

a

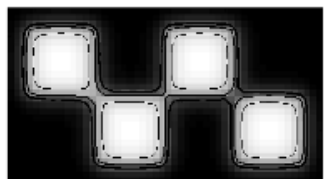

b

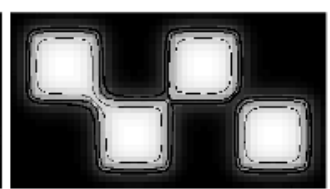

c

Fig. 1. Effects of smoothing: a) original field image, b) result of geometric smoothing, c) result of topological smoothing. The original image is an artificial field for an isocontour halfway between black (0) and white (1). The $0.25,0.5$ and 0.75 isocontours are displayed for $b$ ) and c), with decreasing thickness. Topological smoothing differs from geometric smoothing on saddle regions: likely junctions (left) or cuts (right) are strengthened, undetermined regions are not affected (middle).

\subsection{The Topology Smoothing Algorithm}

If we replace the original values of the scalar field with the topological neighbors, we are not guaranteed to reach a simpler topology. Due to the change of neighbors, some regular points become critical: we then have to iterate the operation to reach a stable regularity. We perform the following relaxation:

$$
f(X) \leftarrow f(X)+\mu \frac{w_{0}\left[f_{0}(X)-f(X)\right]+w_{T}\left[f^{T}(X)-f(X)\right]}{w_{0}+w_{T}}
$$

where $f_{0}(X)$ is the original value of the scalar field, $w_{0}$ and $w_{T}$ the relative weights of the original and the topological value, and $\mu$ the update parameter. $w_{0}, w_{T}, \mu$ are all in $[0,1]$. This algorithm minimizes the following energy function:

$$
E=\sum_{X}\left(w_{0}\left\|f(X)-f_{0}(X)\right\|^{2}+w_{T}\left\|f(X)-f^{T}(X)\right\|\right) .
$$

We have to keep in mind that $f^{T}(X)$ varies whenever $f(X)$ reaches a new topological type: this is why we have to compute an iterative solution rather than a direct solution, that would not account for topology transitions. A low update factor ( $\mu=0.5$ is enough) will also reduce oscillations due to simultaneous topological changes of several neighboring points.

Example: Geometric vs. Topological Smoothing. The algorithm can be used on any scalar field, but only makes sense for fields associated to an isosurface (in 3D) or an isocontour (in 2D). The toy example of Fig. 1 illustrates the effects of topological smoothing on critical points. Compared to traditional geometric smoothing (i.e. using the average value of the neighborhood instead of $f^{T}(X)$; anisotropic or robust smoothing are not considered here for simplicity), topologic smoothing will increase the junctions or the cuts at saddles, depending on initial conditions.

Fig 2 presents a more relevant comparison: a membership function representing gray matter obtained from the segmentation of a noisy $2 \mathrm{D}$ phantom is processed with the topological and geometric smoothing algorithms. Geometric smoothing has been previously observed to simplify the topology of extracted isosurfaces[16]. Our analysis confirms 


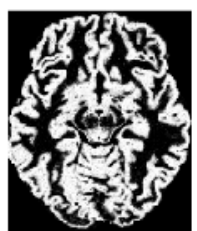

a

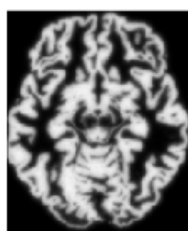

b

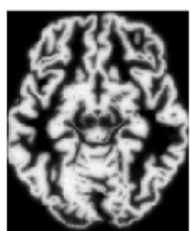

c

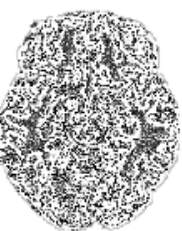

d

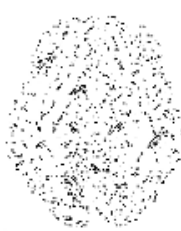

c

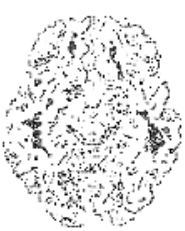

f

Fig. 2. Example on a a segmented phantom: a) original segmented gray matter, b) geometric smoothing, c) topological smoothing. d,e,f) display the critical (black) and extended critical (gray) points for the three images.

that observation: averaging the field over its neighbors are likely to reinforce regular points and remove critical points. However, geometric smoothing makes no distinction between small topological changes (removing the noise) and large ones (affecting the ideal object topology). Topological smoothing will transform the image in a more hierarchical way, preserving and compacting the main topology information, while removing noise-related critical points. The amount of corrected topology is driven by the smoothing factor $w_{T}$; even large critical regions will disappear with repeated iterations, if the factor $w_{T}$ is high enough.

\section{Topology Smoothing in Tissue Classification}

In this section, we show how topological regularization can be incorporated into a segmentation algorithm. Tissue classification techniques often produce on membership or probability functions that represent the likelihood each pixel of the image belongs to a particular tissue class. This function can then used to build an isosurface, e.g. taking the 0.5 isovalue. Topological regularization of this function will therefore improve the topological properties of surfaces derived from the function.

\subsection{Topology-Smoothing Segmentation}

We use a Robust Fuzzy C-means (RFCM) algorithm [11] as the basis for tissue classification. This algorithm imposes a spatial penalty on the standard fuzzy C-means algorithm to improve robustness to noise. The following embedding of topological smoothing applies similarly to expectation-maximization algorithms that perform Gaussian clustering [10]. We integrate the smoothing as an additional step in RFCM:

1. compute the RFCM membership functions $u_{j, k}$ :

$$
u_{j, k}=\frac{\left(\left\|y_{j}-v_{k}\right\|^{2}+\beta \sum_{l \in N_{j}} \sum_{m \neq k} u_{l, m}^{* q}\right)^{1 / q-1}}{\sum_{k}\left(\left\|y_{j}-v_{k}\right\|^{2}+\beta \sum_{l \in N_{j}} \sum_{m \neq k} u_{l, m}^{* q}\right)^{1 / q-1}},
$$


2. relax the membership functions $u_{j, k}^{*}$ toward the topological neighbors $u_{j, k}^{T}$ :

$$
u_{j, k}^{*}=u_{j, k}^{*}+\mu \frac{w_{0}\left[u_{j, k}-u_{j, k}^{*}\right]+w_{T}\left[u_{j, k}^{T}-u_{j, k}^{*}\right]}{w_{0}+w_{T}}
$$

3. compute the RFCM class mean values $v_{k}=\frac{\sum_{j} u_{j, k}^{* q} y_{j}}{\sum_{j} u_{j, k}^{* q}}$.

The membership functions are the topologically smoothed $u_{j, k}^{*}$, but the algorithm is still very similar to the original RFCM algorithm (steps 1 and 3 only). The convergence of the topological smoothing is fast, usually around 20 iterations. The smoothing step only adds one tuning parameter to the algorithm, $w_{T}$ (we set $\mu=0.5, w_{0}=1$ in all cases). Its usual scope is from 0.1 to 1 .

A constraint of segmentation algorithms (FCM or EM-based) is that the membership functions all add to one. When the smoothing is introduced, that property is verified if and only if $\sum_{k} u_{j, k}^{T}=1$. To meet this constraint, the values $u_{j, k}^{T}$ are reprojected using $u_{j, k}^{T}=u_{j, k}^{T} / \sum_{k} u_{j, k}^{T}$. In practice the projection is small, and no significant change has been observed in the result.

\subsection{Over-Smoothing Correction}

An issue that must be addresssed with topological smoothing is the flattening of membership values over small regions. As the smoothing computes a mean of the region, it will lower maxima on thin, elongated regions and spread boundaries between low and high regions. To counteract this effect, we enforce changes only at topologically relevant regions. We used the following property: as long as the corrected value $u_{j, k}^{c}$ stays between the closest inferior and superior values $u_{j, k}^{i n f}, u_{j, k}^{s u p}$ of $u_{j, k}^{*}$, the topological type of the point is preserved. We apply the following correction:

$$
u_{j, k}^{c}=\min \left[\max \left[\left(u_{j, k}^{*}+w_{c} u_{j, k}\right) /\left(1+w_{c}\right), u_{j, k}^{i n f}\right], u_{j, k}^{s u p}\right]
$$

This correction step will guarantee that the smoothed field has values as close to the original data as possible, but with a simplified topology. The parameter $w_{c}$ is not critical, and is set in practice to 0.25 . We also considered using a robust weighted mean for computing $u_{j, k}^{T}$ as an alternative, with similar results.

\subsection{Experimental Evaluation}

The algorithm has been validated on a simulated MR image from the Brainweb database [2], with $3 \%$ and $5 \%$ noise. The segmentation was performed using the FCM, RFCM, topology-smoothing RFCM (T-RFCM), topology-smoothing with correction (TC-RFCM) algorithms, both with $w_{T}=1$. The RFCM parameter has been set to near optimal values, from [11].

We report in Table 2 the misclassification rate (ratio of misclassified pixels over the volume), the RMS error on the membership functions, the ratio of critical points in the volume, the number of handles detected in a connectivity graph (from [13]) for the segmented volume of white matter, and the Euler number of the extracted surface. 
Table 2. Error measures and Topological numbers from Simulated MR results

\begin{tabular}{|l|cc|cc|cc|cc|}
\hline & FCM & & RFCM & \multicolumn{2}{|c|}{ T-RFCM } & \multicolumn{2}{|c|}{ TC-RCM } \\
\hline noise & $3 \%$ & $5 \%$ & $3 \%$ & $5 \%$ & $3 \%$ & $5 \%$ & $3 \%$ & $5 \%$ \\
\hline Misclassification rate (\%) & 3.988 & 6.587 & 3.755 & 4.947 & 4.483 & 5.654 & 4.057 & 5.230 \\
Average RMS error & 0.131 & 0.173 & 0.119 & 0.139 & 0.136 & 0.156 & 0.126 & 0.147 \\
Critical points (\%) & 60.63 & 63.84 & 49.96 & 48.74 & 33.31 & 34.62 & 36.34 & 37.61 \\
Detected handles & 286 & 956 & 129 & 90 & 76 & 48 & 74 & 65 \\
Euler number & -950 & -2750 & -408 & -290 & -268 & -184 & -304 & -240 \\
\hline
\end{tabular}
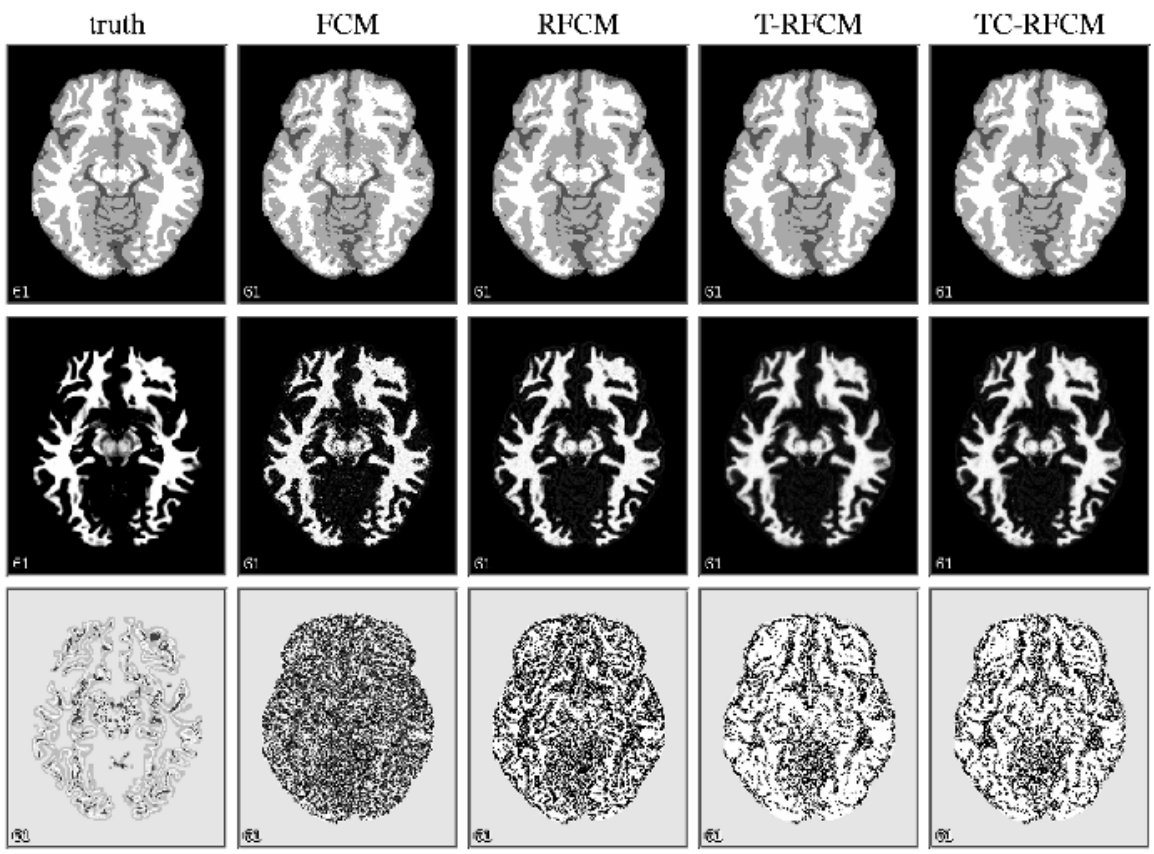

Fig. 3. Results on the simulated MR image with 5\% noise, for the different algorithms: segmentation (top), white matter membership (middle) and corresponding critical points (bottom).

The results show that the topology smoothing is efficient, removing about a third of the critical points and half of the handles. With the correction step, the segmentation errors remain very close to those of RFCM, and are comparable if not better to those of FCM. The membership functions are very similar to RFCM memberships (see Fig.3), free of noise, slightly smoother. The number of critical points is greatly reduced, and they are moved away from the boundaries. The topology is simplified (less handles) and even stabilized (fewer variations with different isovalues) from the smoothing. A posterior correction to reach spherical topology will need to impose fewer modifications of the segmentation, improving accuracy. 


\section{Discussion}

Although many image processing algorithms employ regularization or smoothing to improve robustness to noise, they do not consider how the the topology of the data is affected. We have proposed a smoothing algorithm that directly addresses the topology of scalar fields, and have embedded it into a standard segmentation technique. It makes no assumption on the nature of the object, uses all the information from the image, and avoids hard constraints. Experiments for cortical reconstruction have demonstrated that it imposes minor changes in the overall segmentation, while effectively removing many topology defects. Although our embedding within the segmentation algorithm is currently suboptimal, it should be possible to incorporate topological regularity as a penalty or prior probability function more formally into the optimization.

\section{References}

1. G. Bertrand. Simple points, topological numbers and geodesic neighborhood in cubic grids. Pattern Recognition Letters, 15(10):1003-1011, 1994.

2. D. L. Collins, et al. Design and construction of a realistic digital brain phantom. IEEE Trans. Med.Img., 17(3), 1998.

3. M. Couprie, F. Bezerra, and G. Bertrand. Topological operators for grayscale image processing. J. Electronic Imaging, 10(4):1003-1015, 2001.

4. X. Han, C. Xu, U. Braga-Neto, and J. L. Prince. Topology correction in brain cortex segmentation using a multiscale, graph-based algorithm. IEEE Trans. Med. Img., 21(2):109-121, 2002.

5. X. Han, C. Xu, and J. L. Prince. A topology preserving level set method for geometric deformable models. IEEE Trans. Pat. Analysis and Mach. Intelligence, 25(6):755-768, 2003.

6. J. C. Hart. Morse theory for implicit surface modeling. In H.-C. Hege and K. Polthier, editors, Mathematical Visualization, pp 257-268. Springer-Verlag, Oct. 1998.

7. W. E. Lorensen and H. E. Cline. Marching cubes: A high resolution $3 \mathrm{~d}$ surface construction algorithm. In Proc. SIGGRAPH'87, volume 21, pp 163-169, 1987.

8. J.-F. Mangin, V. Frouin, I. Bloch, J. Regis, and J. Lopez-Krahe. From 3d magnetic resonance images to structural representations of the cortex topography using topology preserving deformations. J. Mathematical Imaging and Vision, 5:297-318, 1995.

9. G. M. Nielson. On marching cubes. IEEE Trans. Visualization and Computer Graphics, 9(3):283-297, 2003.

10. D. Pham, C. Xu, and J. Prince. Current methods in medical image segmentation. In Annual Review of Biomedical Engineering, volume 2 of Annual Reviews, pp 315-337. 2000.

11. D. L. Pham. Spatial models for fuzzy clustering. Computer Vision and Image Understanding, 84:285-297, 2001.

12. F. Segonne, E. Grimson, and B. Fischl. Topological correction of subcortical segmentation. In Proc. MICCAI'03, Montreal, november 2003.

13. D. W. Shattuck and R. M. Leahy. Automated graph-based analysis and correction of cortical volume topology. IEEE Trans. Med. Img., 20(11), 2001.

14. G. H. Weber, G. Scheuermann, and B. Hamann. Detecting critical regions in scalar fields. In Proc. EUROGRAPHICS - IEEE TCVG Symposium on Visualization, Grenoble, may 2003.

15. C. Xu, D. L. Pham, and J. L. Prince. SPIE Handbook on Medical Imaging - Vol. 2, chapter Medical Image Segmentation Using Deformable Models, pp 129-174. SPIE Press, 2000.

16. C. Xu, D. L. Pham, M. E. Rettmann, D. N. Yu, and J. L. Prince. Reconstruction of the human cerebral cortex from magnetic resonance images. IEEE Trans. Med. Img., 18(6), 1999. 